\title{
On the Computational Complexity of the Forcing Chromatic Number
}

\author{
Frank Harary* Wolfgang Slany ${ }^{\dagger}$ Oleg Verbitsky ${ }^{\ddagger}$
}

11 August 2004

\begin{abstract}
We consider vertex colorings of graphs in which adjacent vertices have distinct colors. A graph is $s$-chromatic if it is colorable in $s$ colors and any coloring of it uses at least $s$ colors. The forcing chromatic number $F_{\chi}(G)$ of an $s$-chromatic graph $G$ is the smallest number of vertices which must be colored so that, with the restriction that $s$ colors are used, every remaining vertex has its color determined uniquely. We estimate the computational complexity of $F_{\chi}(G)$ relating it to the complexity class US introduced by Blass and Gurevich. We prove that recognizing if $F_{\chi}(G) \leq 2$ is US-hard with respect to polynomial-time many-one reductions. Moreover, this problem is coNP-hard even under the promises that $F_{\chi}(G) \leq 3$ and $G$ is 3-chromatic. On the other hand, recognizing if $F_{\chi}(G) \leq k$, for each constant $k$, is reducible to a problem in US via disjunctive truth-table reduction.

Similar results are obtained also for forcing variants of the clique and the domination numbers of a graph.
\end{abstract}

\section{Introduction}

The vertex set of a graph $G$ will be denoted by $V(G)$. An $s$-coloring of $G$ is a map from $V(G)$ to $\{1,2, \ldots, s\}$. A coloring $c$ is proper if $c(u) \neq c(v)$ for any adjacent vertices $u$ and $v$. A graph $G$ is $s$-colorable if it has a proper $s$-coloring. The minimum $s$ for which $G$ is s-colorable is called the chromatic number of $G$ and denoted by $\chi(G)$. If $\chi(G)=s$, then $G$ is called s-chromatic.

A partial coloring of $G$ is any map from a subset of $V(G)$ to the set of positive integers. Suppose that $G$ is $s$-chromatic. Let $c$ be a proper $s$-coloring and $p$ be a partial coloring of $G$. We say that $p$ forces $c$ if $c$ is a unique extension of $p$ to a proper $s$-coloring. The domain of $p$ will be called a defining set for $c$. We call $D \subseteq V(G)$ a forcing set in $G$ if this set is defining for some proper s-coloring of $G$.

*Computer Science Department, New Mexico State University, Las Cruces, NM 88003, USA.

${ }^{\dagger}$ Institut für Softwaretechnologie, Technische Universität Graz, A-8010 Graz, Austria.

$\ddagger$ Dept. of Mechanics \& Mathematics, Kyiv University, Ukraine. 
The minimum cardinality of a forcing set is called the forcing chromatic number of $G$ and denoted by $F_{\chi}(G)$.

We study the computational complexity of this graph invariant. To establish the hardness of computing $F_{\chi}(G)$, we focus on the respective slice decision problems which are defined for each non-negative integer $k$ as follows:

$\operatorname{FORCE}_{\chi}(k)$

Given: a graph $G$.

Decide if: $F_{\chi}(G) \leq k$.

The cases of $k=0$ and $k=1$ are tractable. It is clear that $F_{\chi}(G)=0$ iff $\chi(G)=1$, that is, $G$ is empty. Furthermore, $F_{\chi}(G)=1$ iff $\chi(G)=2$ and $G$ is connected, that is, $G$ is a connected bipartite graph. Thus, we can pay attention only to $k \geq 2$. Since there is a simple reduction of $\operatorname{FORCE}_{\chi}(k)$ to $\operatorname{FORCE}_{\chi}(k+1)$ (see Lemma 3.2), it would suffice to show that even $\operatorname{FoRCE}_{\chi}(2)$ is computationally hard. This is indeed the case.

Let 3COL denote the set of 3-colorable graphs and U3COL the set of those graphs in 3COL having a unique, up to renaming colors, proper 3-coloring. First of all, note that a hardness result for $\operatorname{FoRCE}_{\chi}(2)$ is easily derivable from two simple observations:

$$
\begin{aligned}
& \text { If } F_{\chi}(G) \leq 2 \text {, then } G \in 3 \mathrm{COL} \text {; } \\
& \text { If } G \in \mathrm{U} 3 \mathrm{COL} \text {, then } F_{\chi}(G) \leq 2 \text {. }
\end{aligned}
$$

The set 3COL was shown to be NP-complete at the early stage of the NPcompleteness theory in $[31,11]$ by reduction from SAT, the set of satisfiable Boolean formulas. It will be benefittable to use a well-known stronger fact: There is a polynomial-time many-one reduction $p$ from SAT to 3COL which is parsimonious, that is, any Boolean formula $\Phi$ has exactly as many satisfying assignments to variables as the graph $p(\Phi)$ has proper 3-colorings (colorings obtainable from one another by renaming colors are not distinguished). In particular, if $\Phi$ has a unique satisfying assignment, then $p(\Phi) \in \mathrm{U} 3 \mathrm{COL}$ and hence $F_{\chi}(p(\Phi)) \leq 2$, while if $\Phi$ is unsatisfiable, then $p(\Phi) \notin 3 \mathrm{COL}$ and hence $F_{\chi}(p(\Phi))>2$.

Valiant and Vazirani [34] designed a polynomial-time computable randomized transformation $r$ of the set of Boolean formulas such that, if $\Phi$ is a satisfiable formula, then with a non-negligible probability the formula $r(\Phi)$ has a unique satisfying assignment, while if $\Phi$ is unsatisfiable, then $r(\Phi)$ is surely unsatisfiable. Combining $r$ with the parsimonious reduction $p$ of SAT to 3COL, we arrive at the conclusion that $\operatorname{FoRCE}_{\chi}(2)$ is NP-hard with respect to randomized polynomial-time many-one reduction. As a consequence, the forcing chromatic number is not computable in polynomial time unless any problem in NP is solvable by a polynomial-time Monte Carlo algorithm with one-sided error.

We aim at determining the computational complexity of $F_{\chi}(G)$ more precisely. Our first result establishes the hardness of $\operatorname{FonCE}_{\chi}(2)$ with respect to deterministic polynomial-time many-one reduction. The latter reducibility concept will be default in the sequel. The complexity class US, introduced by Blass and Gurevich [2], consists of languages $L$ for which there is a polynomial-time nondeterministic Turing machine $N$ such that a word $x$ belongs to $L$ iff $N$ on input $x$ has exactly one accepting 


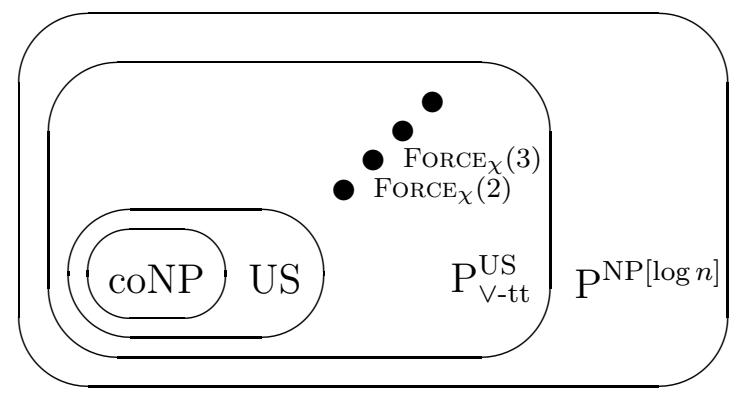

Figure 1: Location of the slice decision problems for $F_{\chi}(G)$ in the hierarchy of complexity classes.

computation path. Denote the set of Boolean formulas with exactly one satisfying assignment by USAT. This set is complete for US. As easily seen, U3COL belongs to US and, by the parsimonious reduction from SAT to 3COL, U3COL is another UScomplete set. By the Valiant-Vazirani reduction, the US-hardness under polynomialtime many-one reduction implies the NP-hardness under randomized reduction and hence the former hardness concept should be considered stronger. It is also known that US includes coNP [2] and this inclusion is proper unless the polynomial time hierarchy collapses [28]. Moreover, the US-hardness implies the coNP-hardness [2]. We prove that the problem $\operatorname{FonCE}_{\chi}(2)$ is US-hard. ${ }^{1}$

On the other hand, we are able to estimate the complexity of all $\operatorname{FORCE}_{\chi}(k)$ from above by putting this family of problems in a complexity class which is a natural extension of US. We show that, for each $k \geq 2$, the problem $\operatorname{ForCE}_{\chi}(k)$ is reducible to a set in US via polynomial-time disjunctive truth-table reduction (see Section 2 for definitions).

Denote the class of decision problems reducible to US under such reductions by $\mathrm{P}_{\mathrm{V} \text {-tt }}^{\mathrm{US}}$. As shown by Chang, Kadin, and Rohatgi [3], $\mathrm{P}_{\mathrm{V} \text {-tt }}^{\mathrm{US}}$ is strictly larger than US unless the polynomial time hierarchy collapses to its third level. Position of the problems under consideration in the hierarchy of complexity classes is shown in Figure 1, where $\mathrm{P}^{\mathrm{NP}[\log n]}$ denotes the class of decision problems solvable by polynomial-time Turing machines with logarithmic number of queries to an NP oracle. The latter class coincides with the class of problems polynomial-time truth-table reducible to NP [17].

Our next result gives a finer information about hardness of $\operatorname{FoRCE}_{\chi}(2)$. Note that, if $\chi(G)=2$, then $F_{\chi}(G)$ is equal to the number of connected components of $G$. It turns out that the knowledge that $\chi(G)=3$ does not help in computing $F_{\chi}(G)$. Moreover, it is hard to recognize whether or not $F_{\chi}(G)=2$ even if it is known that $F_{\chi}(G) \leq 3$. Stating these strengthenings, we relax our hardness concept from the US-hardness to the coNP-hardness (as already mentioned, the former implies the

\footnotetext{
${ }^{1}$ This result is actually equivalent to the reducibility of $\mathrm{U} 3 \mathrm{COL}$ to $\operatorname{FonCE}_{\chi}(2)$. A such reduction would follow from the naive hypothesis, which may be suggested by (1), that a 3-chromatic $G$ is in U3COL iff $F_{\chi}(G)=2$. It should be stressed that the latter is far from being true in view of Lemma 2.7.3 below.
} 


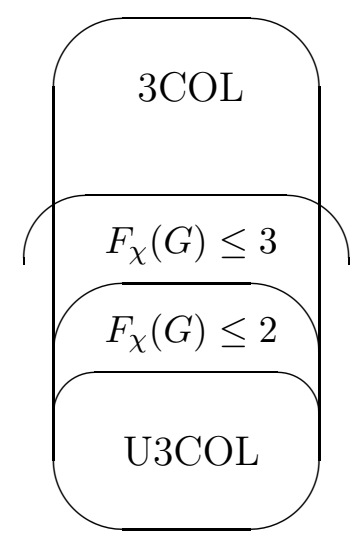

Figure 2: The class $\left\{G: F_{\chi}(G) \leq 2\right\}$ and surroundings.

latter but the converse is not true unless the polynomial time hierarchy collapses). Thus, we prove that the problem $\operatorname{FoRCE}_{\chi}(2)$ is coNP-hard even under the promises that $G \in 3 \mathrm{COL}$ and $F_{\chi}(G) \leq 3$ (see Figure 2). Note that the Valiant-Vazirani reduction implies no kind of a hardness result for the promise version of $\operatorname{FoRCE}_{\chi}(2)$.

In fact, many other graph characteristics also have natural forcing variants. Recall that a clique in a graph is a set of pairwise adjacent vertices. The maximum number of vertices of $G$ in a clique is denoted by $\omega(G)$ and called the clique number of $G$. A clique is optimal if it consists of $\omega(G)$ vertices. A set of vertices is called forcing if it is included into a unique optimal clique. We denote the minimum cardinality of a forcing set by $F_{\omega}(G)$ and call it the forcing clique number of $G$.

Furhtermore, we say that a vertex of a graph $G$ dominates itself and any adjacent vertex. A set $D \subseteq V(G)$ is called dominating if every vertex of $G$ is dominated by a vertex in $D$. The domination number of $G$, denoted by $\gamma(G)$, is the minimum cardinality of a dominating set of $G$. Similarly to the above, a forcing set of vertices is one included into a unique optimal dominating set and the minimum cardinality of a forcing set is denoted by $F_{\gamma}(G)$ and called the forcing domination number of $G$. This graph invariant is introduced and studied by Chartrand, Gavlas, Vandell, and Harary [4].

For the forcing clique and domination numbers we consider the respective slice decision problems $\operatorname{ForCE}_{\omega}(k)$ and $\operatorname{FoRCE}_{\gamma}(k)$ and show the same relation of them to the class US that we have for the forcing chromatic number. Actually, the disjunctive truth-table reducibility to US is proved for all the three numbers by a uniform argument. However, the US-hardness with respect to many-one reductions for $\omega$ and $\gamma$ is proved differently. The case of $\omega$ and $\gamma$ seems combinatorially simpler because of the following equivalence: A graph $G$ has a unique optimal clique iff $F_{\omega}(G)=0$ and similarly with $\gamma$. The study of unique optimum (UO) problems was initiated by Papadimitriou [26]. Due to the US-hardness of the UO CLIQUE and UO DOMINATING SET problems, we are able to show the US-hardness of $\operatorname{FORCE}_{\omega}(k)$ and $\operatorname{FORCE}_{\gamma}(k)$ using only well-known standard reductions, whereas for $\operatorname{FORCE}_{\chi}(k)$ we use somewhat more elaborate reductions involving graph products. 


\section{Overview of previous related work}

Forcing chromatic number of particular graphs. Let $K_{n}$ (resp. $\left.C_{n}, P_{n}\right)$ denote the complete graph (resp. the cycle, the path) on $n$ vertices. As a simple exercise, we have $F_{\chi}\left(C_{2 m+1}\right)=m+1$. In [25] the forcing chromatic number of several Cartesian products is computed: $F_{\chi}\left(C_{2 m+1} \times K_{2}\right)=m+1, F_{\chi}\left(C_{m} \times K_{n}\right)=m(n-3)$ and $F_{\chi}\left(P_{m} \times K_{n}\right)=m(n-3)+2$ if $n \geq 6$, and $F_{\chi}\left(K_{m} \times K_{n}\right)=m(n-m)$ if $n \geq m^{2}$. In [24] a few missing values are determined: $F_{\chi}\left(C_{m} \times K_{3}\right)=\lfloor m / 2\rfloor+1$ and, if $m$ even, $F_{\chi}\left(C_{m} \times K_{5}\right)=2 m$. Our results show that no general approach for efficient computing of the forcing chromatic number is possible unless NP $=\mathrm{P}$ (and even $\mathrm{US}=\mathrm{P})$.

Latin squares and complexity of recognizing a forcing coloring. A Latin square of order $n$ is an $n \times n$ matrix with entries in $\{1,2, \ldots, n\}$ such that every row and column contains all the $n$ numbers. In a partial Latin square some entries may be empty and every number occurs in any row or column at most once. A partial Latin square is called a critical set if it can be completed to a Latin square in a unique way. Colbourn, Colbourn, and Stinson [5] proved that recognition if a given partial Latin square $L$ is a critical set is coNP-hard. Moreover, the problem is coNP-complete even if one extension of $L$ to a Latin square is known.

As it is observed in [25], there is a natural one-to-one correspondence between Latin squares of order $n$ and proper $n$-colorings of the Cartesian square $K_{n} \times K_{n}$ which matches critical sets and forcing colorings. ${ }^{2}$ It follows that it is coNP-hard to recognize if a given partial coloring $p$ of a graph is forcing. Moreover, even if this problem is restricted to graphs $K_{n} \times K_{n}$ and one extension of $p$ to a proper $n$-coloring is given, the problem is coNP-complete (see also Proposition 2.8 below).

Variety of combinatorial forcing numbers. Critical sets are studied since the seventies (see e.g. a survey [21]). The forcing chromatic number (as well as the forcing domination number) attracted attention of researchers in the mid-nineties. In fact, a number of other problems in diverse areas of combinatorics have a similar forcing nature. Not attempting here to give the complete list of such problems and their bibliography, we want to mention at least defining sets in block designs (Gray [12]) and forcing matching number (Harary, Klein, and Živković [16]).

Unique colorability. The concept of a uniquely colorable graph was introduced by Harary, Hedetniemi, and Robinson [15]. Complexity-theoretic concepts related to this combinatorial phenomenon were introduced by Valiant and Vazirani [34] and Blass and Gurevich [2]. However, the complexity-theorists prefer to deal with USAT, the uniqueness version of the archetypical SATisfiability problem. The results established for USAT, as US- and coNP-hardness under many-one reductions and NP-hardness under randomized reductions, carry through for U3COL by means

\footnotetext{
${ }^{2}$ Such a correspondence is also well known for $n$-edge-colorings of the complete bipartite graph $K_{n, n}$.
} 
of the parsimonious many-one reduction of SAT to 3COL. A direct, purely combinatorial way to show the coNP-hardness of U3COL is given by a result of Greenwell and Lovász ([13], see also [19, Theorem 8.5]). They prove, in particular, that if a connected graph $G$ is not 3-colorable then the categorical product $G \cdot K_{3}$ is a uniquely colorable graph. On the other hand, if $G$ is 3 -colorable then $G \cdot K_{3}$ can be colored in two different ways. The latter follows from a simple observation: any proper 3-coloring of one of the factors efficiently induces a proper 3-coloring of product $G_{1} \cdot G_{2}$. By the NP-completeness of 3COL, we arrive at the conclusion that the problem of recognizing, given a graph $H$ and its proper 3-coloring, whether or not $H$ is uniquely 3-colorable, is coNP-complete. Later this complexity-theoretic fact was observed by Dailey [8] who, though does not refer to [13], uses the identical combinatorial argument.

\section{Organization of the paper}

In Section 2 we define the categorical and the Cartesian graph products, which will play an important role in our proofs, and prove some preliminary lemmas about forcing sets in product graphs. We also state a few basic bounds for $F_{\chi}(G)$ and determine the complexity of recognition if a given set of vertices in a graph is forcing (the latter results is not used in the sequel but is worth being noticed). The hardness of $\operatorname{ForcE}_{\chi}(k)$ is established in Section 3. A closer look at $\operatorname{ForCE}_{\chi}(2)$ is taken in Section 4. In Section 5 we analyze the complexity of a related graph invariant, namely, the largest cardinality of an inclusion-minimal forcing set. Before taking into consideration the forcing clique and domination numbers, we suggest a general setting for forcing combinatorial numbers in Section 6. It is built upon the standard formal concept of an NP optimization problem. We benefit from this formal framework in some proofs. Section 7 is devoted to the forcing clique and domination numbers. The disjunctive reducibility of $\operatorname{FoncE}_{\pi}(k)$ to US for $\pi \in\{\chi, \omega, \gamma\}$ is shown in Section 8. Section 9 contains a concluding discussion and some open questions.

\section{Background}

\subsection{Basics of complexity theory}

We suppose that the discrete structures under consideration are encoded by binary words. For example, graphs are naturally representible by their adjacency matrices. The set of all binary words is denoted by $\{0,1\}^{*}$. A decision problem is identified with a language, i.e. subset of $\{0,1\}^{*}$, consisting of all yes-instances. A many-one reduction of a problem $X$ to a problem $Y$ is a map $r:\{0,1\}^{*} \rightarrow\{0,1\}^{*}$ such that $x \in X$ iff $r(x) \in Y$. If $r(x)$ is computable in time bounded by a polynomial in the length of $x$, the reduction is called polynomial-time. We write $X \leq_{\mathrm{m}}^{\mathrm{P}} Y$ to say that there is a polynomial-time many-one reduction from $X$ to $Y$.

Let $C$ be a class of decision problems (or languages). A problem $Z$ is called $C$-hard if any $X$ in $C$ is $\leq_{\mathrm{m}}^{\mathrm{P}}$-reducible to $Z$. A problem $Z$ is called $Z$-complete if 
$Z$ is $C$-hard and belongs to $C$. USAT and U3COL are examples of US-complete problems.

A disjunctive truth-table reduction of a language $X$ to a language $Y$ is a transformation which takes any word $x$ to a set of words $y_{1}, \ldots, y_{m}$ so that $x \in X$ iff $y_{i} \in Y$ for at least one $i \leq m$. We write $X \leq_{\mathrm{v} \text {-tt }}^{\mathrm{P}} Y$ to say that there is such a polynomial-time reduction from $X$ to $Y$.

If $\mathrm{C}$ is a class of languages and $\leq$ is a reducibility, then $\mathrm{C} \leq X$ means that $Y \leq X$ for all $Y$ in $\mathrm{C}$ (i.e. $X$ is C-hard under $\leq$ ) and $X \leq \mathrm{C}$ means that $X \leq Y$ for some $Y$ in $\mathrm{C}$.

The formal framework of promise problems is developed in [30]. Let $Y$ and $Q$ be languages. Whenever referring to a decision problem $Y$ under the promise $Q$, we mean that membership in $Y$ is to be decided only for inputs in $Q$. A reduction $r$ of an (ordinary) decision problem $X$ to a problem $Y$ under the promise $Q$ is a usual many-one reduction form $X$ to $Y$ with the additional requirement that $r(x) \in Q$ for all $x$. This definition allows us to extend the notion of $C$-hardness to promise problems.

A polynomial-time computable function $h:\{0,1\}^{*} \times\{0,1\}^{*} \rightarrow\{0,1\}^{*}$ is called an $A N D_{2}$ function for a language $Z$ if for any pair $x, y$ we have both $x$ and $y$ in $Z$ iff $h(x, y)$ is in $Z$. A such $h$ is an $O R_{2}$ function for $Z$ if we have at least one of $x$ and $y$ in $Z$ iff $h(x, y)$ is in $Z$.

\subsection{Graph products}

Let $E(G)$ denote the set of edges of a graph $G$. Given two graphs $G_{1}$ and $G_{2}$, we define a product graph on the vertex set $V\left(G_{1}\right) \times V\left(G_{2}\right)$ in two ways. Vertices $\left(u_{1}, u_{2}\right)$ and $\left(v_{1}, v_{2}\right)$ are adjacent in the Cartesian product $G_{1} \times G_{2}$ if either $u_{1}=v_{1}$ and $\left\{u_{2}, v_{2}\right\} \in E\left(G_{2}\right)$ or $u_{2}=v_{2}$ and $\left\{u_{1}, v_{1}\right\} \in E\left(G_{1}\right)$. They are adjacent in the categorical product $G_{1} \cdot G_{2}$ if both $\left\{u_{1}, v_{1}\right\} \in E\left(G_{1}\right)$ and $\left\{u_{2}, v_{2}\right\} \in E\left(G_{2}\right)$.

A set $V\left(G_{1}\right) \times\{v\}$ for each $v \in V\left(G_{2}\right)$ will be called a $G_{1}$-layer of $v$ and a set $\{u\} \times V\left(G_{2}\right)$ for each $u \in V\left(G_{1}\right)$ will be called a $G_{2}$-layer of $u$.

Lemma 2.1 (Sabidussi, see [19, Theorem 8.1]) $\chi(G \times H)=\max \{\chi(G), \chi(H)\}$.

If $c$ is a proper coloring of $G$, it is easy to see that $c^{*}(x, y)=c(x)$ is a proper coloring of $G \cdot H$. We will say that $c$ induces $c^{*}$. Similarly, any proper coloring of $H$ induces a proper coloring of $G \cdot H$. This implies the following well-known fact.

Lemma $2.2 \chi(G \cdot H) \leq \min \{\chi(G), \chi(H)\}$.

The next proposition shows that the Cartesian and the categorical products are, respectively, $\mathrm{AND}_{2}$ and $\mathrm{OR}_{2}$ functions for $3 \mathrm{COL}$ (see [22] for an exposition of $\mathrm{AND}$ and OR functions).

Proposition 2.3 (.1) $G \times H \in 3 \mathrm{COL}$ if both $G$ and $H$ are in 3COL.

(.2) $G \cdot H \in 3 \mathrm{COL}$ if at least one of $G$ and $H$ is in 3COL. 
Proof. Item 1 is straightforward by Lemma 2.1. However, Item 2 does not follow solely from Lemma 2.2 because the equality $\chi(G \cdot H)=\min \{\chi(G), \chi(H)\}$ is the unproven Hedetniemi conjecture. Luckily, the conjecture is known to be true for 4-chromatic graphs (El-Zahar and Sauer, see [19, Section 8.2]), and this suffices for our claim.

\subsection{Preliminary lemmas}

Lemma 2.4 (Greenwell-Lovász [13]) Let $G$ be a connected graph with $\chi(G)>n$. Then $G \cdot K_{n}$ is uniquely $n$-colorable.

The proof can be found also in [19, Theorem 8.5]. We will use not only Lemma 2.4 itself but also a component of its proof stated as the next lemma. We call a coloring injective if it is an injective map.

Lemma 2.5 Let $G$ be a connected graph and $p$ be an injective coloring of a $K_{n}$-layer of $G \cdot K_{n}$. Then p forces the $K_{n}$-induced coloring of $G \cdot K_{n}$.

Proof. Suppose that $p$ is an injective coloring of a $K_{n}$-layer of $u \in V(G)$ and that a proper $n$-coloring $c$ of $G \cdot K_{n}$ extends $p$. Let $v \in V(G)$ be adjacent to $u$. Inferably, $c(v, i)=c(u, i)$ for any $i \in V\left(K_{n}\right)$. Since $G$ is connected, $c$ is forced by $p$ to be monochrome on each $G$-layer.

Lemma 2.6 Any proper 3-coloring of $K_{3} \cdot K_{3}$ is induced by one of the two factors $K_{3}$.

Proof. Let $V\left(K_{3}\right)=\{1,2,3\}$ and denote $L_{i}=\{(i, 1),(i, 2),(i, 3)\}$. Suppose that $c$ is a coloring of $K_{3} \cdot K_{3}$. Consider it on one of the layers, say, $L_{2}$. If $\left|c\left(L_{2}\right)\right|=3$, then $c$ is induced by the second factor on the account of Lemma 2.5. Assume that $\left|c\left(L_{2}\right)\right|=$ 2 , for example, $c(2,1)=1, c(2,2)=2$, and $c(2,3)=2$. Then $c(1,2)=c(3,3)=3$ is forced, contradictory to the fact that these vertices are adjacent. There remains the possibility that $\left|c\left(L_{2}\right)\right|=1$, for example, $c(2,1)=c(2,2)=c(2,3)=2$. Color 2 can occur neither in $L_{1}$ nor in $L_{3}$. Assume that one of these layers has both colors 1 and 3 , say, $c(1,1)=1$ and $c(1,2)=3$. This forces $c(3,3)=2$, a contradiction. Thus, $|c(L)|=1$ is possible only if $L_{1}, L_{2}$, and $L_{3}$ are all monochrome and have pairwise distinct colors. This is the coloring induced by the first factor.

\subsection{A few basic bounds}

We call two $s$-colorings equivalent if they are obtainable from one another by permutation of colors. Proper $s$-colorings of a graph $G$ are equivalent if they determine the same partition of $V(G)$ into $s$ independent sets. Let $N_{\chi}(G)$ denote the number of such partitions for $s=\chi(G)$. Thus, $N_{\chi}(G)$ is equal to the number of inequivalent proper $s$-colorings of $s$-chromatic $G$, while the total number of such colorings is equal to $\chi(G) ! N_{\chi}(G)$. A graph $G$ is uniquely colorable if $N_{\chi}(G)=1$. In particular, $G \in \mathrm{U} 3 \mathrm{COL}$ iff $\chi(G)=3$ and $N_{\chi}(G)=1$. 


\section{Lemma 2.7}

(.1) $\chi(G)-1 \leq F_{\chi}(G) \leq \log _{2} N_{\chi}(G)+\log _{2}(\chi(G) !)$.

(.2) If $N_{\chi}(G)=1$, then $F_{\chi}(G)=\chi(G)-1$.

(.3) For all $n=4 k+2$, there are 3-chromatic graphs $G_{k}$ with $F_{\chi}\left(G_{k}\right)=2$ and $N_{\chi}\left(G_{k}\right)=2^{k-1}+1$.

The lower bound in Item 1 is sharp by Item 2. The upper bound is sharp because, for example, $F_{\chi}\left(m K_{2}\right)=m$ while $N_{\chi}\left(m K_{2}\right)=2^{m-1}$, where $m K_{2}$ denotes the graph consisting of $m$ isolated edges. Item 3 shows that the converse of Item 2 is false. It is also worth noting that both the lower and the upper bounds in Item 1 are computationally hard, even if one is content with finding an approximate value. The NP-hardness of approximation of the chromatic number is established in [23] (see also [9] for the best of subsequent results). A hardness result for approximate computing $\log _{2} N_{\chi}(G)$, even for 3-chromatic graphs, is obtained in [35].

Proof. Item 2 and the lower bound in Item 1 are obvious. To prove the upper bound in Item 1 , we have to show that a $k$-chromatic graph $G$ has a forcing set of at most $l=\left\lfloor\log _{2}\left(k ! N_{\chi}(G)\right)\right\rfloor$ vertices $v_{1}, v_{2}, \ldots$ Let $C_{1}$ be the set of all $k ! N_{\chi}(G)$ proper $k$-colorings of $G$. We choose vertices $v_{1}, v_{2}, \ldots$ one by one as follows. Let $i \geq 1$. Assume that the preceding $i-1$ vertices have been chosen and that a set of colorings $C_{i}$ has been defined and has at least 2 elements. We set $v_{i}$ to be an arbitrary vertex such that not all colorings in $C_{i}$ coincide at $v_{i}$. Furthermore, we assign $v_{i}$ a color $p\left(v_{i}\right)$ occurring in the multiset $\left\{c\left(v_{i}\right): c \in C_{i}\right\}$ least frequently. Finally, we define $C_{i+1}=\left\{c \in C_{i}: c\left(v_{i}\right)=p\left(v_{i}\right)\right\}$. Note that $\left|C_{i+1}\right| \leq\left|C_{i}\right| / 2$. We eventually have $\left|C_{i+1}\right|=1$ for some $i \leq l=\left\lfloor\log _{2}\left|C_{1}\right|\right\rfloor$. For this $i$, denote the single coloring in $C_{i+1}$ by $c$. By construction, $v_{1}, \ldots, v_{i}$ is defining for $c$.

To prove Item 3, consider $H=K_{3} \times K_{2}$. This graph has two inequivalent colorings $c_{1}$ and $c_{2}$ shown in Figure 3. Let $u, v, w \in V(H)$ be as in Figure 3. Note that a partial coloring $p_{1}(u) \neq p_{1}(v)$ forces $c_{1}$ or its equivalent and that $p_{2}(u)=$ $p_{2}(v) \neq p_{2}(w)$ forces $c_{2}$.

Let $G_{k}$ consist of $k$ copies of $H$ with all $u$ and all $v$ identified, that is, $G_{k}$ has $4 k+2$ vertices. Since the set $\{u, v\}$ stays forcing in $G_{k}$, we have $F_{\chi}\left(G_{k}\right)=2$. If $u$ and $v$ are assigned the same color, we are free to assign each copy of $w$ any of the two remaining colors. It follows that $N_{\chi}\left(G_{k}\right)=2^{k-1}+1$.

\subsection{Complexity of a forcing set recognition}

Proposition 2.8 The problem of recognizing, given a graph $G$ and two vertices $u, v \in V(G)$, whether or not $\{u, v\}$ is a forcing set is US-complete.

Proof. Suppose that $\chi(G) \geq 3$ for else the question is efficiently decidable. Set $p(u)=1$ and $p(v)=2$. It is easy to see that $\{u, v\}$ is a forcing set iff $p$ forces a proper 3-coloring of $G$. Verification of the latter condition is clearly in US. 

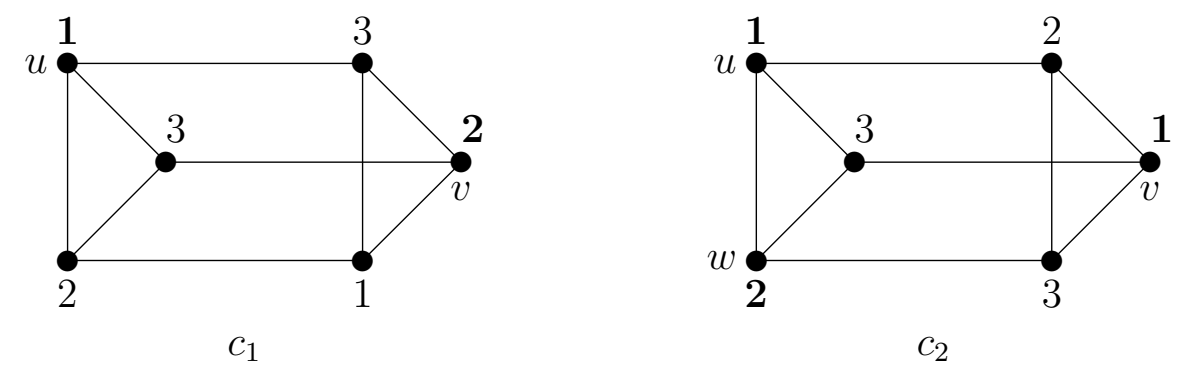

Figure 3: Proper 3-colorings of $K_{3} \times K_{2}$.

We now describe a reduction $R$ of the US-complete problem U3COL to the problem under consideration. If an input graph $G$ is empty, let $R(G)$ be an arbitrary graph with two vertices which are not a forcing set. Otherwise, let $u$ and $v$ be the lexicographically first pair of adjacent vertices in $G$. We set $R(G)=(G, u, v)$. It is not hard to see that $G \in \mathrm{U} 3 \mathrm{COL}$ iff $R(G)$ consists of a graph and a 2-vertex forcing set in it.

\section{Complexity of $F_{\chi}(G)$ : A lower bound}

Theorem 3.1 For each $k \geq 2$, the problem FORCE $_{\chi}(k)$ is US-hard. Moreover, this holds true even if we consider only connected graphs.

We first observe that the family of problems $\operatorname{FORCE}_{\chi}(k)$ is linearly ordered with respect to the $\leq_{\mathrm{m}}^{\mathrm{P}}$-reducibility. A simple reduction showing this does not preserve connectedness of graphs. However, if we restrict ourselves to connected graphs, we are able to show that $\operatorname{FoRCE}_{\chi}(2)$ remains the minimum element in this order. Afterwards we prove that $\operatorname{FORCE}_{\chi}(2)$ is US-hard (even for connected graphs).

Lemma 3.2 $\operatorname{FoRCE}_{\chi}(k) \leq_{\mathrm{m}}^{\mathrm{P}} \operatorname{FoRCE}_{\chi}(k+1)$.

Proof. Given a non-empty graph $G$, we add one isolated vertex to it. Denoting the result by $G+K_{1}$, it is enough to notice that $F_{\chi}\left(G+K_{1}\right)=F_{\chi}(G)+1$.

Lemma 3.3 Let $k \geq 2$. Then $\operatorname{FonCE}_{\chi}(2)$ reduces to $\operatorname{FonCE}_{\chi}(k)$ even if we consider the problems only for connected graphs.

Proof. Let $G$ be a graph on $n$ vertices and $m \leq n$. Writing $H=G \oplus m K_{2}$, we mean that

- $V(H)=\left\{v_{1}, \ldots, v_{n}\right\} \cup \bigcup_{i=1}^{m}\left\{a_{i}, b_{i}\right\}$,

- $H$ induces on $\left\{v_{1}, \ldots, v_{n}\right\}$ a graph isomorphic to $G$,

- $\left\{v_{i}, a_{i}\right\}$ and $\left\{a_{i}, b_{i}\right\}$ for all $i \leq m$ are edges of $H$, and

- $H$ has no other edges. 


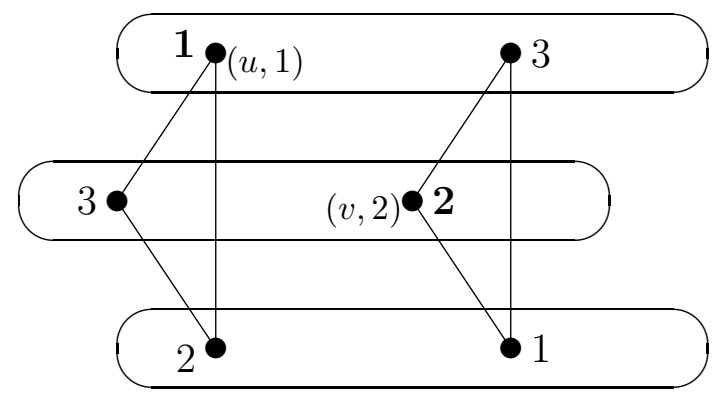

Figure 4: Proof of Lemma 3.5 (Case 1).

Suppose that $\chi(G) \geq 3$ and $H=G \oplus m K_{2}$. Let us check that $F_{\chi}(H) \leq 2+m$ if $F_{\chi}(G)=2$ and $F_{\chi}(H) \geq 3+m$ if $F_{\chi}(G) \geq 3$. This will give us the following reduction of $\operatorname{ForcE}_{\chi}(2)$ to $\operatorname{ForCE}_{\chi}(k)$ : Given $G$, construct an $H=G \oplus(k-2) K_{2}$.

Let $F_{\chi}(G)=2$. Note that $G$ must be 3 -chromatic. To show that $F_{\chi}(H) \leq 2+m$, we construct a forcing set of $2+m$ vertices. We will identify $G$ and its copy spanned by $\left\{v_{1}, \ldots, v_{n}\right\}$ in $H$. Let $v_{j}$ and $v_{l}$ be two vertices such that a partial coloring $p\left(v_{j}\right)=1$ and $p\left(v_{l}\right)=2$ forces a proper 3-coloring $c$ of $G$. Color each $b_{i}$ differently from $c\left(v_{i}\right)$. Then $\left\{v_{j}, v_{l}, b_{i}, \ldots, b_{m}\right\}$ becomes a forcing set in $H$.

Let $F_{\chi}(G) \geq 3$. Clearly, any forcing set contains all $b_{i}$ 's. We hence have to show that no set $S=\left\{b_{1}, \ldots, b_{m}, x, y\right\} \subset V(H)$ is forcing in $H$. We will assume that $\chi(G)=3$ for else the claim is easy. Suppose that $x \in\left\{v_{j}, a_{j}\right\}, y \in\left\{v_{l}, a_{l}\right\}$, and $S$ is augmented with a coloring $p$ extendable to a proper 3-coloring $c$ of $H$. Let $c^{\prime}$ denote the restriction of $c$ to $\left\{v_{1}, \ldots, v_{n}\right\}$. Since $c^{\prime}$ is not a unique coloring of $G$ compatible with $p$, there is another such coloring $c^{\prime \prime}$. Obviously, $c^{\prime \prime}$ and $p$ have a common extension to a proper coloring of $H$. Thus, $S$ is not a forcing set.

Lemma 3.4 $\operatorname{FORCE}_{\chi}(2)$ is US-hard even if restricted to connected graphs.

To prove the lemma, we proceed by reduction from U3COL. Note that U3COL remains US-complete when restricted to connected graphs and that our reduction will preserve connectedness. Since the class of 2-colorable graphs is tractable and can be excluded from consideration, the desired reduction is given by the following lemma.

Lemma 3.5 Suppose that $\chi(G) \geq 3$. Then $G \in \mathrm{U} 3 \mathrm{COL}$ iff $F_{\chi}\left(G \times K_{3}\right)=2$.

Proof. Case 1: $G \in \mathrm{U} 3 \mathrm{COL}$. We have to show that $F_{\chi}\left(G \times K_{3}\right)=2$.

Fix arbitrary $u, v \in V(G)$ whose colors in the proper 3-coloring of $G$ are different, for example, $u$ and $v$ can be any adjacent vertices of $G$. Let $V\left(K_{3}\right)=\{1,2,3\}$. Assign $p(u, 1)=1$ and $p(v, 2)=2$ and check that $p$ forces a proper 3 -coloring of $G \times K_{3}$. Assume that $c$ is a proper 3-coloring of $G \times K_{3}$ consistent with $p$. Since $c$ on each $G$-layer coincides with the 3 -coloring of $G$ up to permutation of colors, we easily infer that $c(v, 1)=c(u, 2)=3$ (see Figure 4 ). This implies $c(u, 3)=2$ and 
$c(v, 3)=1$. Thus, in each $G$-layer we have two vertices with distinct colors which determines colors of all the other vertices. As easily seen, the coloring obtained is really proper.

Case 2: $G \in 3 \mathrm{COL} \backslash \mathrm{U} 3 \mathrm{COL}$. We have to check that $F_{\chi}\left(G \times K_{3}\right) \geq 3$.

Given a partial coloring $p$ of two vertices $a, b \in V\left(G \times K_{3}\right)$, we have to show that it is not forcing. The cases that $p(a)=p(b)$ or that $a$ and $b$ are in the same $G$ or $K_{3}$-layer are easy. Without loss of generality we therefore suppose that $p(a)=1$, $p(b)=2, a=(u, 1)$, and $b=(v, 2)$, where $u$ and $v$ are distinct vertices of $G$. Define two partial colorings of $G$ by $c_{1}(u)=c_{1}(v)=1$ and by $c_{2}(u)=1, c_{2}(v)=3$.

Subcase 2.1: Both $c_{1}$ and $c_{2}$ extend to proper 3-colorings of $G$. Denote the extensions by $e_{1}$ and $e_{2}$ respectively. Denote the three $G$-layers of $G \times K_{3}$ by $G_{1}, G_{2}, G_{3}$ and consider $e_{1}, e_{2}$ on $G_{1}$. For each $i=1,2, e_{i}$ and $p$ agree and have a common extension to a proper coloring of $G \times K_{3}$. Thus, $p$ is not forcing.

Subcase 2.2: Only $c_{1}$ extends to a proper 3-coloring of $G$. Since $G$ is not uniquely colorable, there must be at least two extensions, $e_{1}$ and $e_{2}$, of $c_{1}$ to proper 3 -colorings of $G$. As in the preceding case, $e_{1}$ and $e_{2}$ each agree with $p$ and together with $p$ extend two distinct colorings of $G \times K_{3}$.

Subcase 2.3: Only $c_{2}$ extends to a proper coloring of $G$. This case is completely similar to Subcase 2.2.

Case 3: $G \notin 3 \mathrm{COL}$. We have $\chi\left(G \times K_{3}\right) \geq 4$ by Lemma 2.1 and $F_{\chi}\left(G \times K_{3}\right) \geq 3$ by Lemma 2.7.1.

Theorem 3.1 immediately follows from Lemmas 3.4 and 3.3.

\section{Hardness of $\operatorname{ForCE}_{\chi}(2)$ : A closer look}

Theorem 4.1 The problem FORCE $_{\chi}(2)$ is coNP-hard even under the promises that $F_{\chi}(G) \leq 3$ and $\chi(G) \leq 3$ and even if an input graph $G$ is given together with its proper 3-coloring.

Let us for a while omit the promise that $F_{\chi}(G) \leq 3$. Then the theorem is provable by combining the Greenwell-Lovász reduction of coNP to US (Lemma 2.4) and our reduction of US to $\operatorname{FoRCE}_{\chi}(2)$ (Lemma 3.5). Doing so, we easily deduce the following:

- If $\chi(G)>3$, then $G \cdot K_{3}$ is uniquely 3 -colorable and hence $F_{\chi}\left(\left(G \cdot K_{3}\right) \times K_{3}\right)=2$.

- If $\chi(G)=3$, then $G \cdot K_{3}$ is non-uniquely 3 -colorable and hence $F_{\chi}\left(\left(G \cdot K_{3}\right) \times\right.$ $\left.K_{3}\right) \geq 3$.

To obtain Theorem 4.1 (the weakened version without the promise $F_{\chi}(G) \leq 3$ ), it now suffices to make the following observation.

Lemma $4.2 \chi\left(\left(G \cdot K_{3}\right) \times K_{3}\right)=3$ for any graph $G$. Moreover, a proper 3-coloring is efficiently obtainable from the explicit product representation of $\left(G \cdot K_{3}\right) \times K_{3}$. 


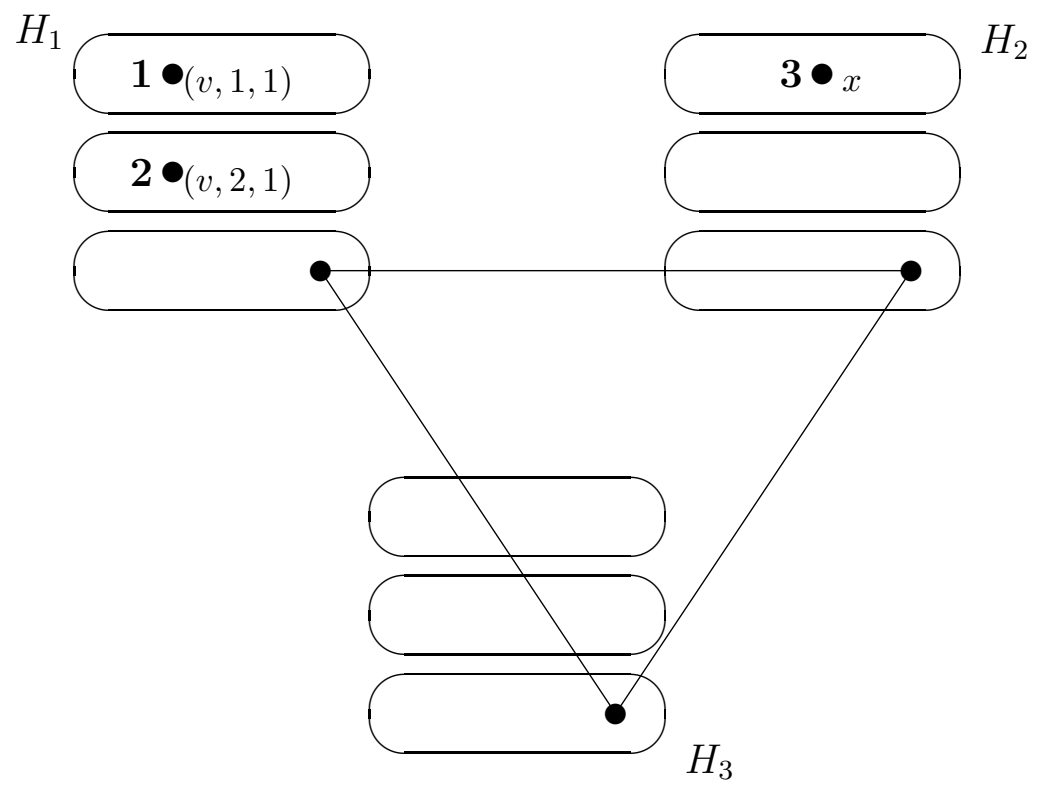

Figure 5: $\left(G \cdot K_{3}\right) \times K_{3}($ Proof of Lemma 4.3)

Proof. By Lemma 2.2, we have $\chi\left(G \cdot K_{3}\right) \leq 3$ and hence, by Lemma 2.1, $\chi((G$. $\left.\left.K_{3}\right) \times K_{3}\right)=3$. Let $V\left(K_{3}\right)=\{1,2,3\}$ and denote $V_{i, j}=\{(v, i, j): v \in V(G)\}$. It is not hard to see that $V_{1,1} \cup V_{2,2} \cup V_{3,3}, V_{1,2} \cup V_{2,3} \cup V_{3,1}$, and $V_{1,3} \cup V_{2,1} \cup V_{3,2}$ is a partition of $V\left(\left(G \cdot K_{3}\right) \times K_{3}\right)$ into independent sets.

To obtain the full version of Theorem 4.1, we only slightly modify the reduction: Before transforming $G$ in $\left(G \cdot K_{3}\right) \times K_{3}$, we add to $G$ a triangle with one vertex in $V(G)$ and two other vertices new. Provided $\chi(G) \geq 3$, this does not change $\chi(G)$ and hence the modified transformation is an equally good reduction. The strengthening (the promise $F_{\chi}(G) \leq 3$ ) is given by the following lemma.

Lemma 4.3 If a graph $G$ is connected and contains a triangle, then $F_{\chi}\left(\left(G \cdot K_{3}\right) \times\right.$ $\left.K_{3}\right) \leq 3$.

Proof. Let $v$ be a vertex of a triangle $T$ in $G$. Consider the product $H=G \cdot K_{3}$ and a partial coloring $p(v, 1)=1, p(v, 2)=2$. We claim that $p$ forces the $K_{3}$-induced coloring of $H$. Obviously, the latter is an extension of $p$. To show that no other extension is possible, assume that $c$ is a proper 3-coloring of $H$ compatible with $p$ and consider the restriction of $c$ on $T \cdot K_{3}$. By Lemma 2.6, $c$ on $T \cdot K_{3}$ coincides with the coloring induced by the second factor. In particular, $c(v, 3)=3$. Our claim now follows from the connectedness of $G$ by Lemma 2.5 .

Now, let $H_{1}, H_{2}, H_{3}$ denote the three $H$-layers in $H \times K_{3}$ and, for each $i=1,2,3$, let $G_{i 1}, G_{i 2}, G_{i 3}$ denote the three $G$-layers in $H_{i}$. Let $p(v, 1,1)=1, p(v, 2,1)=2$ be the forcing partial coloring for $H_{1}$ as described above (see Figure 5). Thus, $p$ forces coloring the whole $G_{1 j}$ in color $j$ for each $j=1,2,3$. Suppose that $c$ is a proper 3-coloring of $H \times K_{3}$ that agrees with $p$. From the product structure of $H \times K_{3}$ we 
see that, for each $j$, in $G_{2 j}$ there cannot occur color $j$. Let $T^{2}$ denote the copy of $T \cdot K_{3}$ in $H_{2}$. By Lemma 2.6, $c$ on $T^{2}$ is induced by the second factor and hence $c(v, 1,2), c(v, 2,2)$, and $c(v, 3,2)$ are pairwise distinct. By Lemma 2.5, each of $G_{21}$, $G_{22}$, and $G_{23}$ is monochrome and these layers receive pairwise distinct colors. We already know that $c\left(G_{2 j}\right) \neq j$. Thus, when we define $p$ in the third point by $p(x)=3$ for an arbitrary $x \in G_{21}$, this forces $c\left(G_{21}\right)=3, c\left(G_{22}\right)=1$, and $c\left(G_{23}\right)=2$. Since every vertex in $G_{3 j}$ is in triangle with a vertex in $G_{1 j}$ and a vertex in $G_{2 j}, c$ is uniquely extrapolated on $\mathrm{H}_{3}$.

The proof of Theorem 4.1 is complete.

\section{Maximum size of a minimal forcing set}

Another related invariant of a graph $G$ is the largest cardinality of an inclusionminimal forcing set in $G$. We will denote this number by $F_{\chi}^{*}(G)$. A complexity analysis of $F_{\chi}^{*}(G)$ is easier owing to the characterization of uniquely colorable graphs obtained in [14].

Lemma 5.1 (Hajiabolhassan, Mehrabadi, Tusserkani, and Zaker [14]) $A$ connected graph $G$ is uniquely 3-colorable iff $F_{\chi}^{*}(G)=2$.

Theorem 5.2 The problem of deciding, given a graph $G$ and its proper 3-coloring, whether or not $F_{\chi}^{*}(G) \leq 2$ is coNP-complete.

Proof. The problem is in coNP because a no-instance of it has a certificate consisting of a proper 3-coloring $c$ of $G$ and a 3-vertex set $A \subset V(G)$ such that no 2-element subset $B$ of $A$ is defining for $c$. The latter fact, for each $B$, is certified by another proper 3-coloring $c_{B}$ that agrees with $c$ on $B$ but differs from $c$ somewhere outside $B$.

The completeness is proved by reduction from the decision problem whether or not $\chi(G)>3$. The latter problem is coNP-complete even if restricted to connected graphs with $\chi(G) \geq 3$. Let $G$ be a such graph. Our reduction just transforms $G$ into the categorical product $G \cdot K_{3}$. If $\chi(G)>3$, then $G \cdot K_{3}$ is uniquely 3-colorable by Lemma 2.4 and, by Lemma 5.1 , we have $F_{\chi}^{*}\left(G \cdot K_{3}\right)=2$. If $\chi(G)=3$, then $G \cdot K_{3}$ has at least two inequivalent proper 3-colorings, namely, those induced by the two factors. By Lemma 5.1, we have $F_{\chi}^{*}\left(G \cdot K_{3}\right) \geq 3$.

\section{General setting}

In fact, many other graph characteristics also have natural forcing variants. Taking those into consideration, it will be convenient to use the formal concept of an NP optimization problem (see e.g. [6]).

Let $\{0,1\}^{*}$ denote the set of binary strings. The length of a string $w \in\{0,1\}^{*}$ is denoted by $|w|$. We will use notation $[n]=\{1,2, \ldots, n\}$.

An NP optimization problem $\pi=\left(\mathrm{opt}_{\pi}, I_{\pi}, \mathrm{sol}_{\pi}, \mathrm{v}_{\pi}\right)$ (where subscript $\pi$ may be omitted) consists of the following components. 
- opt $\in\{\max , \min \}$ is a type of the problem.

- $I \subseteq\{0,1\}^{*}$ is the polynomial-time decidable set of instances of $\pi$.

- Given $x \in I$, we have $\operatorname{sol}(x) \subset\{0,1\}^{*}$, the set of feasible solutions of $\pi$ on instance $x$. We suppose that all $y \in \operatorname{sol}(x)$ have the same length that depends only on $|x|$ and is bounded by $|x|^{O(1)}$. Given $x$ and $y$, it is decidable in polynomial time whether $y \in \operatorname{sol}(x)$.

- $v:\{0,1\}^{*} \times\{0,1\}^{*} \rightarrow \mathbf{N}$ is a polynomial-time computable objective function taking on positive integer values. If $y \in \operatorname{sol}(x)$, then $v(x, y)$ is called the value of $y$.

The problem is, given an instance $x$, to compute the optimum value

$$
\pi(x)=\operatorname{opt}_{y \in \operatorname{sol}(x)} v(x, y) \text {. }
$$

A such problem is called polynomially bounded if $v(x, y)=|x|^{O(1)}$ for all $x \in I$ and $y \in \operatorname{sol}(x)$.

Any $y \in \operatorname{sol}(x)$ whose value is optimum is called an optimum solution of $\pi$ on instance $x$. Let $\operatorname{optsol}(x)$ denote the set of all such $y$. Given an NP optimization problem $\pi$, we define

$$
\mathrm{UO}_{\pi}=\{x:|\operatorname{optsol}(x)|=1\} .
$$

Example 6.1 The problem of computing the chromating number of a graph is expressible as a quadruple $\chi=(\min , I$, sol, $v)$ as follows. A graph $G$ with vertex set $V(G)=\left\{v_{1}, \ldots, v_{n}\right\}$ is represented by its adjacency matrix written down in line row by row as a binary string $x$ of length $n^{2}$. A feasible solution, that is a proper coloring $c: V(G) \rightarrow[n]$, is represented by a binary string $y=c\left(v_{1}\right) \ldots c\left(v_{n}\right)$ of length $n^{2}$, where a color $i$ is encoded by string $0^{i-1} 10^{n-i}$. The value $v(x, y)$ is equal to the actual number of colors occurring in $y$.

Example 6.2 For the problem of computing the clique number it is natural to fix the following representation. An instance graph $G$ is encoded as above. A feasible solution, which is a subset of $V(G)$, is encoded by its characteristic binary string of length $n$. The problem of computing the domination number is represented in the same way.

Given a non-empty set $U \subseteq\{0,1\}^{l}$, we define force $(U)$ to be the minimum cardinality of a set $S \subseteq[l]$ such that there is exactly one string in $U$ with 1 at every position from $S$. With each NP optimization problem $\pi$ we associate its forcing number $F_{\pi}$, an integer-valued function of instances of $\pi$ defined by

$$
F_{\pi}(x)=\operatorname{force}(\operatorname{optsol}(x)) .
$$

Let $\operatorname{FoRCE}_{\pi}(k)=\left\{x: F_{\pi}(x) \leq k\right\}$. It is easy to check that, if $\chi, \omega$, and $\gamma$ are represented as in Examples 6.1 and 6.2 , then $F_{\chi}, F_{\omega}$, and $F_{\gamma}$ are precisely those graph invariants introduced in Section 1. 
Note that $\operatorname{force}(U)=0$ iff $U$ is a singleton. It follows that for $\pi \in\{\omega, \gamma\}$ we have

$$
x \in \mathrm{UO}_{\pi} \text { iff } F_{\pi}(x)=0 .
$$

This will be the starting point of our analysis of decision problems $\operatorname{FORCE}_{\omega}(k)$ and $\operatorname{FORCE}_{\gamma}(k)$ in the next section.

\section{Hardness of $\operatorname{ForCE}_{\omega}(k)$ and $\operatorname{FonCE}_{\gamma}(k)$}

Lemma 7.1 (Thierauf [32, Section 3.2.3]) The decision problem $\mathrm{UO}_{\omega}$ is UShard.

As [32] does not give a detailed proof of this fact, for the reader's convenience we prove Lemma 7.1 in Appendix. The next lemma follows from a few well-known standard reductions and hence its proof is also moved to Appendix.

Lemma 7.2 $\mathrm{UO}_{\omega} \leq_{\mathrm{m}}^{\mathrm{P}} \mathrm{UO}_{\gamma}$.

Thus, $\mathrm{UO}_{\omega}$ and $\mathrm{UO}_{\gamma}$ are both US-hard.

Lemma 7.3 Let $\pi \in\{\omega, \gamma\}$. Then $\operatorname{FonCE}_{\pi}(k) \leq_{\mathrm{m}}^{\mathrm{P}} \operatorname{FoRCE}_{\pi}(k+1)$ for any $k \geq 0$.

Proof. Given a graph $G$, we have to construct a graph $H$ such that $F_{\pi}(G) \leq k$ iff $F_{\pi}(H) \leq k+1$. It suffices to ensure that

$$
F_{\pi}(H)=F_{\pi}(G)+1 .
$$

Let $\pi=\omega$. Let $H$ be the result of adding to $G$ two new vertices $u$ and $v$ and the edges $\{w, u\}$ and $\{w, v\}$ for all $w \in V(G)$. Any optimal clique in $H$ consists of an optimal clique in $G$ and of either $u$ or $v$. Hence any forcing set in $H$ consists of a forcing set in $G$ and of either $u$ or $v$ (we use the terminology of Section 1). This implies (3).

If $\pi=\gamma$, we obtain $H$ from $G$ by adding a new isolated edge.

Putting it all together, we make the following conclusion.

Theorem 7.4 Let $\pi \in\{\omega, \gamma\}$. Then

$$
\mathrm{US} \leq_{\mathrm{m}}^{\mathrm{P}} \mathrm{UO}_{\pi}=\operatorname{FORCE}_{\pi}(0) \leq_{\mathrm{m}}^{\mathrm{P}} \operatorname{FORCE}_{\pi}(k) \leq_{\mathrm{m}}^{\mathrm{P}} \operatorname{FORCE}_{\pi}(k+1)
$$

for any $k \geq 0$. 


\section{Complexity of $\operatorname{FORCE}_{\pi}(k)$ : An upper bound}

We first state a simple general property of the class US.

Lemma 8.1 Every US-complete set has an $A N D_{2}$ function. ${ }^{3}$

Proof. It suffices to prove the lemma for any particular US-complete set, for example, USAT. Given two Boolean formulas $\Phi$ and $\Psi$, rename the variables in $\Psi$ so the formulas become over disjoint sets of variables and consider the conjunction $\Phi \wedge \Psi$. As easily seen, the conjunction is in USAT iff both $\Phi$ and $\Psi$ are in USAT.

In Section 6 with a non-empty set $U \subseteq\{0,1\}^{l}$ we associated the number force $(U)$. Additionally, let us put force $(\emptyset)=\infty$.

Theorem 8.2 Let $\pi$ be a polynomially bounded NP optimization problem. Then $\operatorname{FORCE}_{\pi}(k) \leq_{\mathrm{V} \text {-tt }}^{\mathrm{P}}$ US for each $k \geq 0$.

Proof. We will assume that $\pi$ is a minimization problem (the case of maximization problems is quite similar). Suppose that $v(x, y) \leq|x|^{c}$ for a constant $c$. Given $1 \leq m \leq|x|^{c}$, we define

$$
\operatorname{sol}^{m}(x)=\{y \in \operatorname{sol}(x): v(x, y)=m\}
$$

and

$$
F_{\pi}^{m}(x)=\operatorname{force}\left(\operatorname{sol}^{m}(x)\right) .
$$

In particular, $F_{\pi}^{m}(x)=F_{\pi}(x)$ if $m=\pi(x)$.

Let $k$ be a fixed integer. Notice that

$$
F_{\pi}(x) \leq k \text { iff } \bigvee_{m=1}^{|x|^{c}}\left(F_{\pi}^{m}(x) \leq k \wedge \pi(x) \geq m\right)
$$

(actually, only the disjunction member for $m=\pi(x)$ has chance to be true). The set of pairs $(x, m)$ with $\pi(x) \geq m$ is in coNP and hence in US. Let us now show that the set of $(x, m)$ with $F_{\pi}^{m}(x) \leq k$ is disjunctively reducible to US.

Recall that $\operatorname{sol}(x) \subseteq\{0,1\}^{l(x)}$, where $l(x) \leq|x|^{d}$ for a constant $d$. Define $T$ to be the set of quadruples $(x, m, l, D)$ such that $m$ and $l$ are positive integers, $D \subseteq[l]$, and there is a unique $y \in \operatorname{sol}^{m}(x)$ of length $l$ with all 1's in positions from $D$. It is easy to see that $T$ is in US and

$$
F_{\pi}^{m}(x) \leq k \text { iff } \bigvee_{\substack{l, D: l \leq|x|^{d} \\ D \subseteq[l],|D| \leq k}}(x, m, l, D) \in T
$$

Combining this equivalence with (4), we conclude that $F_{\pi}(x) \leq k$ iff there are numbers $m \leq|x|^{c}$ and $l \leq|x|^{d}$ and a set $D \subseteq[l]$ of size at most $k$ such that

$$
(x, m, l, D) \in T \wedge \pi(x) \geq m \text {. }
$$

\footnotetext{
${ }^{3}$ In fact, a stronger fact is true: every US-complete set has an AND function of unbounded arity.
} 
By Lemma 8.1, this conjunction is expressible as a proposition about membership of the quadruple $(x, m, l, D)$ in a US-complete set. Thus, the condition $F_{\pi}(x) \leq k$ is equivalent to a disjunction of less than $|x|^{c+d(k+1)}$ propositions each verifiable in US.

Corollary 8.3 Let $\pi \in\{\chi, \omega, \gamma\}$. Then $\operatorname{FORCE}_{\pi}(k) \leq_{\mathrm{V} \text {-tt }}^{\mathrm{P}}$ US for each $k \geq 0$.

\section{Concluding discussion and open questions}

1. We have considered forcing versions of three popular graph invariants: the chromatic, the clique, and the domination numbers $\left(F_{\chi}, F_{\omega}\right.$, and $F_{\gamma}$ respectively). We have shown that slice decision problems for each of $F_{\chi}, F_{\omega}$, and $F_{\gamma}$ are as hard as US under the many-one reducibility and as easy as US under the disjunctive truth-table reducibility. The latter upper bound is actually true for the forcing variant of any polynomially bounded NP optimization problem. The lower bound in the case of $F_{\omega}$ and $F_{\gamma}$ is provable by using standard reductions on the account of a close connection with the unique optimum problems $\mathrm{UO}_{\omega}$ and $\mathrm{UO}_{\gamma}$. However, in the case of $F_{\chi}$ we use somewhat more elaborate reductions involving graph products. We point out two simple reasons for the distinction between $F_{\chi}$ and $F_{\omega}, F_{\gamma}$. First, unlike the case of $\omega$ and $\gamma$, the unique colorability of a graph is apparently inexpressible in terms of $F_{\chi}$ (cf. Lemma 2.7.3). Second, we currently do not know any relation between $F_{\chi}, F_{\omega}$, and $F_{\gamma}$ as optimization problems (cf. the subsequent discussion).

2. As well known, most NP-complete decision problems, including those for $\chi, \omega$, and $\gamma$, are rather similar to each other: There are parsimonious many-one reductions among them. However, it is also well known that the total similarity disappears as soon as we get interested in approximation properties of the underlying NP optimization problems or in parametrized complexity of the decision problems themselves. In particular, $\chi, \omega$, and $\gamma$ occupy different positions in the two mentioned hierarchies. It would be interesting to compare complexities of $F_{\chi}, F_{\omega}$, and $F_{\gamma}$.

The first question is which reducibility concept is here most appropriate. The strongest one seems to be as follows: Given NP optimization problems $\pi$ and $\varpi$, let $\pi \prec \varpi$ if there is a polynomial-time transformation $r: I_{\pi} \rightarrow I_{\varpi}$ such that $F_{\pi}(x)=$ $F_{\varpi}(r(x))$. As the simplest example, note that $\omega$ and $\alpha$ are $\prec$-equivalent, where $\alpha$ denotes the independence number of a graph (however, the standard reduction from $\alpha$ to $\tau$, the vertex cover number of a graph, though combinatorially simple, is not a $\prec$-reduction). If NP optimization problems $\pi$ and $\varpi$ have property (2), then $\mathrm{UO}_{\pi} \leq_{\mathrm{m}}^{\mathrm{P}} \mathrm{UO}_{\varpi}$ whenever $\pi \prec \varpi$. However, the reduction proving Lemma 7.2 is not a $\prec$-reduction from $\omega$ to $\gamma$. Thus, in order to find relations between various forcing nunbers, one has to more elaborate on reductions and perhaps even use weaker reduction concepts.

3. Our results imply that the problems $\operatorname{FonCE}_{\pi}(k)$ for any $\pi \in\{\chi, \omega, \gamma\}$ and $k \geq 0$ (except $k=0,1$ if $\pi=\chi$ ) have the same polynomial-time Turing degree. Let $\operatorname{FoncE}_{\pi}(*)=\left\{(x, k): F_{\pi}(x) \leq k\right\}$. How related are $\operatorname{FoncE}_{\pi}(*)$ for $\pi \in\{\chi, \omega, \gamma\}$ 
under $\leq_{\mathrm{m}}^{\mathrm{P}}$-reductions (and even $\leq_{\mathrm{T}}^{\mathrm{P}}$-reductions)? Note that these three decision problems are US-hard and belong to $\Sigma_{2}^{\mathrm{P}}$ (see [29] for an overview of the latter complexity class).

4. Is $\operatorname{FORCE}_{\pi}(*)$ NP-hard under $\leq_{\mathrm{m}}^{\mathrm{P}}$-reductions for any $\pi$ under consideration?

5. Let UCOL be the set of all uniquely colorable graphs (with no restriction on the chromatic number). Is it true that $\mathrm{UCOL} \leq_{\mathrm{m}}^{\mathrm{P}} \operatorname{FORCE}_{\chi}(2)$ ? Note that UCOL $\neq$ $\mathrm{UO}_{\chi}$ if the latter set is understood in the formal sense of Section 6. It is not hard to show that UCOL is US-hard.

6. Theorem 4.1 estimates the relative complexity of the class of graphs with forcing chromatic number 2 with respect to the wider classes shown in Figure 2. The picture will be complete if we can estimate complexity of U3COL under the promise that $F_{\chi}(G)=2$.

7. Is the forcing chromatic number hard to approximate within a constant factor?

8. Given a graph with perfect matching, Harary, Klein, and Živković [16] define its forcing matching number as the minimum size of a forcing set of edges, where the latter is a set contained in a unique perfect matching. Is this graph invariant computationally hard? Note that, despite computing the number of perfect matchings is as hard as computing the number of proper 3-colorings [33], uniqueness of a perfect matching is efficiently decidable [10, 18]. Note also that forcing sets of edges are efficiently recognizable and, as a consequence, for each fixed $k$ the problem of deciding if the forcing matching number does not exceed $k$ is polynomial-time solvable.

\section{Acknowledgement}

This work was initiated when the third author visited the Department of Information Systems at the Vienna University of Technology. He sincerely thanks Georg Gottlob for his hospitality during this visit.

\section{References}

[1] A.V. Aho, J.E. Hopcroft, J.D. Ullman. The design and analysis of computer algorithms. Addison-Wesley, 1976.

[2] A. Blass, Y. Gurevich. On the unique satisfiability problem. Information and Control 55:80-88 (1982).

[3] R. Chang, J. Kadin, P. Rohatgi. On unique satisfiability and the threshold behaviour of randomized reductions. Journal of Computer and System Sciences 50(3):359-373 (1995).

[4] G. Chartrand, H. Gavlas, R.C. Vandell, F. Harary. The forcing domination number of a graph. J. Comb. Math. Comb. Comput. 25:161-174 (1997).

[5] C.J. Colbourn, M.J. Colbourn, D.R. Stinson. The computational complexity of recognizing critical sets. Graph theory, Proc. 1-st Southeast Asian Colloq. Singapore 1983, Lect. Notes Math., Vol. 1073, 248-253 (1984). 
[6] P. Crescenzi. A short quide to approximation preserving reductions. In: Proc. of the 12-th Ann. Conference on Computational Complexity, 262-272 (1997).

[7] P. Crescenzi, C. Fiorini, R. Silvestri. A note on approximation of the MAX CLIQUE problem. Information Processing Letters 40:1-5 (1991).

[8] D.P. Dailey. Uniqueness of colorability and colorability of planar 4-regular graphs are NP-complete. Discrete Math. 30, 289-293 (1980).

[9] U. Feige, J. Kilian. Zero knowledge and the chromatic number. Journal of Computer and System Sciences 57(2):187-199 (1998).

[10] H.N. Gabow, H. Kaplan, R.E. Tarjan. Unique maximum matching algorithms. In: Proc. of the 31-st Ann. Symp. on Theory of Comp. 70-78 (1999).

[11] M.R. Garey, D.S. Johnson, L.J. Stockmeyer. Some simplified NP-complete graph problems. Theor. Comp. Sci. 1:237-267 (1976).

[12] K. Gray. On the minimum number of blocks defining a design. Bull. Aust. Math. Soc. 41(1)97-112 (1990).

[13] D.L. Greenwell, L. Lovász. Applications of product colouring. Acta Math. Acad. Sci. Hung. 25:335-340 (1974).

[14] H. Hajiabolhassan, M.L. Mehrabadi, R. Tusserkani, M. Zaker. A characterization of uniquely vertex colorable graphs using minimal defining sets. Discrete Math. 199:233236 (1999).

[15] F. Harary, S.T. Hedetniemi, R.W. Robinson. Uniquely colorable graphs. J. Combin. Theory 6:264-270 (1969); 9:221 (1970).

[16] F. Harary, D. Klein, T. Živković. Graphical properties of polyhexes: Perfect matching vector and forcing. J. Math. Chemistry 6:295-306 (1991).

[17] L. Hemachandra. Structure of complexity classes: separations, collapses, and completeness. In: Proc. of the MFCS. Lecture Notes in Computer Science, Vol. 324, 59-73 (1988).

[18] T.M. Hoang, M. Mahajan, T. Thierauf. Unique perfect matching is in NC. Submitted (2004).

[19] W. Imrich, S. Klavzar. Product graphs. Structure and recognition. Wiley-Interscience Series in Discrete Mathematics and Optimization. Wiley, Chichester (2000).

[20] V. Kann. On the approximability of NP-complete optimization problems. Ph.D. thesis. Royal Institute of Technology, Stockholm, 1992.

[21] A.D. Keedwell. Critical sets for Latin squares, graphs, and block designs: a survey. Congr. Numer. 113:231-245 (1996).

[22] J. Köbler, U. Schöning, and J. Torán. The Graph Isomorphism problem: its structural complexity. Birkhäuser, 1993.

[23] C. Lund, M. Yannakakis. On the hardness of approximating minimization problems. J. of the ACM 41(5):960-981 (1994).

[24] M. Mahdian, E.S. Mahmoodian, R. Naserasr, F. Harary. On defining sets of vertex colorings of the Cartesian product of a cycle with a complete graph. In: Combinatorics, Graph Theory, and Algorithms, Y. Alavi, D.R. Lick, and A. Schwenk Eds. 461-467. New Issues Press, Kalamazoo (1999). 
[25] E.S. Mahmoodian, R. Naserasr, M. Zaker. Defining sets in vertex colorings of graphs and Latin rectangles. Discrete Math. 167-168:451-460 (1997).

[26] C. Papadimitriou. On the complexity of unique solutions. J. of the ACM 31(2):392400 (1984).

[27] C. Papadimitriou. Computational complexity. Addison-Wesley, 1994.

[28] D. Ranjan, S. Chari, and P. Rohtagi. Improving known solutions is hard. In: Proc. of the 18-th ICALP. Lecture Notes in Computer Science, Vol. 510, 381-392 (1991).

[29] M. Schaefer. Graph Ramsey theory and the polynomial hierarchy. In: Proc. of the 31-st Ann. ACM Symp. on Theory of Computing, 592-601 (1999).

[30] A.L. Selman. Promise problems and complexity classes. Information and Computation 78:87-98 (1988).

[31] L.J. Stockmeyer. Planar 3-colorability is NP-complete. SIGACT News 5(3):19-25 (1973).

[32] T. Thierauf. The computational complexity of equivalence and isomorphism problems. Lecture Notes in Computer Science, Vol. 1852. Springer (2000).

[33] L.G. Valiant. The complexity of computing the permanent. Theor. Comp. Sci. 8:189201 (1979).

[34] L.G. Valiant, V.V. Vazirani. NP is as easy as detecting unique solution. Theor. Comp. Sci. 47(1):287-300 (1986).

[35] D. Zuckerman. On unapproximable versions of NP-complete problems. SIAM J. Comput. 25(6):1293-1304 (1996).

\section{A US-hardness of $\mathrm{UO}_{\omega}$ and $\mathrm{UO}_{\gamma}$}

We here prove Lemmas 7.1 and 7.2. We will make use of some reducibility concepts for NP optimizations problems.

Let $\pi$ and $\varpi$ be NP optimization problems of the same type. Let $f:\{0,1\}^{*} \rightarrow\{0,1\}^{*}$ and $g:\{0,1\}^{*} \times\{0,1\}^{*} \rightarrow\{0,1\}^{*}$ be polynomial-time computable functions such that for every $x \in I_{\pi}$ we have $f(x) \in I_{\varpi}$ and for every $y \in \operatorname{sol}_{\varpi}(f(x))$ we have $g(x, y) \in \operatorname{sol}_{\pi}(x)$. Such a pair $(f, g)$ is said to be an $S$-reduction from $\pi$ to $\varpi$ if for every $x \in I_{\pi}$ we have

$$
\operatorname{opt}_{\pi}(x)=\operatorname{opt}_{\varpi}(f(x))
$$

and, in addition, for every $y \in \operatorname{sol}_{\varpi}(f(x))$ we have

$$
\mathrm{v}_{\pi}(x, g(x, y))=\mathrm{v}_{\varpi}(f(x), y) .
$$

We call an $S$-reduction $(f, g)$ a parsimonious reduction from $\pi$ to $\varpi$ if, for any $x \in I_{\pi}$, $g(x, \cdot)$ is a one-to-one map from $\operatorname{sol}_{\varpi}(f(x))$ onto $\operatorname{sol}_{\pi}(x)$. If only a weaker condition is met, namely, that $g(x, \cdot)$ is a one-to-one correspondence between the optimum solutions of $\varpi$ on instance $f(x)$ and the optimum solutions of $\pi$ on instance $x$, then $(f, g)$ will be called a weakly parsimonious reduction from $\pi$ to $\varpi$.

Given a Boolean formula $\Phi$ in the conjunctive normal form (CNF), let $\sigma(\Phi)$ denote the maximum number of clauses of $\Phi$ satisfiable by the same truth assignment to the variables. By $\sigma_{3}$ we denote the restriction of $\sigma$ to $3 \mathrm{CNF}$ formulas (those having at most 3 literals per 
a clause). We regards $\sigma$ and $\sigma_{3}$ as NP optimization problems. Both the problems belong to the class MAX NP introduced by Papadimitriou and Yannakakis. Crescenzi, Fiorini, and Silvestri [7], who introduced the notion of an S-reduction, proved that every problem in MAX NP is $S$-reducible to $\omega$. We need a somewhat stronger fact about $\sigma_{3}$.

Lemma A.1 There is a parsimonuios reduction from $\sigma_{3}$ to $\omega$.

Proof. Let $\phi$ be a disjunctive clause and $X$ be the set of variables occurring in $\phi$. Let $D(\phi)$ denote the set of all conjunctions that contain every variable from $X$ or its negation and imply $\phi$. Note that $\phi$ is logically equivalent to the disjunction of all $\psi$ in $D(\phi)$.

Given a $3 \mathrm{CNF}$ formula $\Phi$, we construct a graph $G$ as follows. Let $V(G)$ be the union of $D(\phi)$ over all clauses $\phi$ of $\Phi$. We join $\psi_{1}$ and $\psi_{2}$ in $V(G)$ by an edge if these conjunctions are consistent, i.e., no variable occurring in $\psi_{1}$ occurs in $\psi_{2}$ with negation and vice versa.

Proof of Lemma 7.1. Denote the restrictions of SAT and USAT to 3CNF formulas by 3SAT and U3SAT respectively. Since there is a parsimonious $\leq_{\mathrm{m}}^{\mathrm{P}}$-reduction from SAT to 3SAT (see e.g. [27]), U3SAT is US-complete. We now show that U3SAT $\leq_{\mathrm{m}}^{\mathrm{P}} \mathrm{UO}_{\omega}$.

Given a 3CNF formula $\Phi$, let $G$ be the graph constructed from $\Phi$ by the reduction of Lemma A.1. Let $m$ denote the number of clauses in $\Phi$ and $H=G+2 K_{m-1}$, the disjont union of $G$ and two copies of $K_{m-1}$.

If $\Phi \in \mathrm{U} 3 \mathrm{SAT}$, then $\omega(H)=\omega(G)=m$ and $H \in \mathrm{UO}_{\omega}$ because $G \in \mathrm{UO}_{\omega}$.

If $\Phi \in \mathrm{SAT} \backslash \mathrm{U} 3 \mathrm{SAT}$, then $\omega(H)=\omega(G)=m$ and $H \notin \mathrm{UO}_{\omega}$ because $G \notin \mathrm{UO}_{\omega}$.

If $\Phi \notin \mathrm{SAT}$, then $\omega(H) \leq m-1$ and $H \notin \mathrm{UO}_{\omega}$ having at least two optimal cliques.

Thus, $\Phi \in \mathrm{U} 3 \mathrm{SAT}$ iff $H \in \mathrm{UO}_{\omega}$.

Proof of Lemma 7.2. Recall that a vertex cover of a graph $G$ is a set $S \subseteq V(G)$ such that every edge of $G$ is incident to a vertex in $S$. The vertex cover number of $G$ is defined to be the minimum cardinality of a vertex cover of $G$ and denoted by $\tau(G)$. It is easy to see and well known that $S \subseteq V(G)$ is a clique in $G$ iff $V(G) \backslash S$ is a vertex cover in the graph complementary to $G$. It follows that

$$
\mathrm{UO}_{\omega} \leq_{\mathrm{m}}^{\mathrm{P}} \mathrm{UO}_{\tau}
$$

We now show that

$$
\mathrm{UO}_{\tau} \leq_{\mathrm{m}}^{\mathrm{P}} \mathrm{UO}_{\gamma}
$$

by regarding $\tau$ and $\gamma$ as NP minimization problems and designing a weakly parsimonious reduction from $\tau$ to $\gamma$. Recall that, given a set $X$ and a system of its subsets $\mathcal{Y}=$ $\left\{Y_{1}, \ldots, Y_{n}\right\}$, a subsystem $\left\{Y_{i_{1}}, \ldots, Y_{i_{k}}\right\}$ is called a set cover if $X=\bigcup_{j=1}^{k} Y_{i_{j}}$. We compose two known reductions between minimization problems, Reduction A from the minimum vertex cover to the minimum set cover ([1, Theorem 10.11]) and Reduction B from the minimum set cover to the minimum domination number (an adaptation of $[20$, Theorem A.1]).

Reduction $A$. Given a graph $G$ and its vertex $v$, let $I(v)$ denote the set of the edges of $G$ incident to $v$. Consider the set $X=E(G)$ and the system of its subsets $\mathcal{Y}=$ $\{I(v): v \in V(G)\}$. Then $S \subseteq V(G)$ is an optimal vertex cover for $G$ iff $\{I(v): v \in S\}$ is an optimal set cover for $(X, \mathcal{Y})$.

Reduction $B$. Given a set $X=\left\{x_{1}, \ldots, x_{m}\right\}$ and a system of sets $\mathcal{Y}=\left\{Y_{1}, \ldots, Y_{n}\right\}$ such that $X=\bigcup_{j=1}^{n} Y_{j}$, we construct a graph $H$ as follows. $V(H)$ contains each element $x_{i}$ in duplicate, namely, $x_{i}$ itself and its clone $x_{i}^{\prime}$. There is no edge between these $2 m$ vertices. 
Other vertices of $H$ are indices $1, \ldots, n$, with all possible $\left(\begin{array}{l}n \\ 2\end{array}\right)$ edges between them. If and only if $x_{i} \in Y_{j}$, both $x_{i}$ and $x_{i}^{\prime}$ are adjacent to $j$. There are no more vertices and edges.

Observe that any optimal dominating set $D \subset V(H)$ is included in $[n]$. Indeed, if $D$ contains both $x_{i}$ and $x_{i}^{\prime}$, then it can be reduced by replacing these two vertices by only one vertex $j$ such that $x_{i} \in Y_{j}$. If $D$ contains exactly one of $x_{i}$ and $x_{i}^{\prime}$, say $x_{i}$, then it should contain some $j$ such that $x_{i} \in Y_{j}$ to dominate $x_{i}^{\prime}$. But then $D$ can be reduced just by removing $x_{i}$.

It is also clear that a set $D \subseteq[n]$ is dominating in $H$ iff $\left\{Y_{j}: j \in D\right\}$ is a set cover for $(X, \mathcal{Y})$. Thus, there is a one-to-one correspondence between optimal set covers for $(X, \mathcal{Y})$ and optimal dominating sets in $H$.

Remark A.2 Using (5) and Theorem 8.2, we can easily show that for the vertex cover number $\tau$ we also have

$$
\mathrm{US} \leq_{\mathrm{m}}^{\mathrm{P}} \mathrm{UO}_{\tau}=\operatorname{FORCE}_{\tau}(0) \leq_{\mathrm{m}}^{\mathrm{P}} \operatorname{FORCE}_{\tau}(k) \leq_{\mathrm{m}}^{\mathrm{P}} \operatorname{FORCE}_{\tau}(k) \leq_{\mathrm{V}-\mathrm{tt}}^{\mathrm{P}} \mathrm{US} .
$$

